(2) Open Access Full Text Article

\title{
Development of docetaxel nanocrystals surface modified with transferrin for tumor targeting
}

This article was published in the following Dove Press journal:

Drug Design, Development and Therapy

16 December 2016

Number of times this article has been viewed

Jin-Seok Choi

Jeong-Sook Park

College of Pharmacy, Institute of Drug Research and Development, Chungnam National University, Yuseong-gu, Daejeon, South Korea

Correspondence: Jin-Seok Choi, Jeong-Sook Park

College of Pharmacy, Institute of Drug Research and Development, Chungnam National University, 99 Daehak-ro, Yuseong-gu, Daejeon 34I34, South Korea $\mathrm{Tel}+82428217323 ;+82428215932$

Fax +82428236566

Email c3428I@gmail.com;

eicosa@cnu.ac.kr

\begin{abstract}
The purpose of this study was to develop the surface modification of docetaxel nanocrystals (DTX-NCs) with apo-Transferrin human (Tf) for improving the cellular uptake and cytotoxicity of DTX. DTX-NCs were prepared by a nanoprecipitation method, and the surface modified with Tf by an adsorption method (Tf-DTX-NCs). The morphology and particle size of DTX-NCs and Tf-DTX-NCs were characterized using a field emission scanning electron microscope and zetasizer. An in vitro drug release study was performed in phosphate-buffered saline containing $0.5 \%(\mathrm{w} / \mathrm{v})$ Tween 80 for 24 hours. Cellular uptake was studied at $0.5,1$, and 2 hours. A cytotoxicity study was performed using the A549 (human lung cancer) cell line after 24-, 48-, and 72-hour treatments. The mean sizes were $295 \pm 97$ and $398 \pm 102 \mathrm{~nm}$ for DTX-NCs and Tf-DTX-NCs, respectively. Tf-DTX-NCs and DTX-NCs exhibited rapid drug release, whereas DTX (pure) was slowly released. Tf-DTX-NCs showed higher cellular uptake than DTX-NCs in confocal microscopic and quantitative studies. Moreover, at DTX concentration of $100 \mu \mathrm{g} / \mathrm{mL}$, Tf-DTX-NCs $(82.6 \% \pm 0.8 \%)$ showed higher cytotoxicity than DTX-NCs $(77.4 \% \pm 4.1 \%$ ) and DTX (pure; $20.1 \% \pm 4.6 \%$ ) for 72 -hour treatment. In conclusion, Tf-DTXNCs significantly improved the cellular uptake and cytotoxicity of DTX in the A549 cell line.

Keywords: docetaxel, nanocrystals, surface modification, apo-Transferrin human
\end{abstract}

\section{Introduction}

Chemotherapy often results in increased side effects owing to the nonspecificity of chemotherapeutic drugs. Many researchers have studied nanoparticles (NPs) for tumor targeting. ${ }^{1-4}$ NPs can accumulate in tumor sites by the enhanced permeability and retention (EPR) effect. ${ }^{5,6}$ Therefore, drug-loaded NPs show high accumulation in tumor sites. In recent years, many studies have evaluated the targeting ability of NPs by using in vivo imaging techniques. ${ }^{7-10}$

Various types of NPs have been developed for drug delivery, including polymeric NPs, ${ }^{11}$ solid lipid NPs (SLN), ${ }^{12}$ liposome, ${ }^{13}$ self-assembly NPs, ${ }^{14}$ and drug nanocrystals (NCs). ${ }^{15}$ NPs can be surface modified using targeting ligands, such as transferrin, ${ }^{16}$ hyaluronic acid, ${ }^{17}$ trastuzumab, ${ }^{18}$ and peptides, ${ }^{19}$ to improve their tumor targeting and cellular internalization.

Docetaxel (DTX) belongs to the taxane class of drugs, which also includes paclitaxel (PTX). The structure of DTX is similar to that of PTX. Clinically, DTX is used to treat many types of cancers such as breast cancer, ovarian cancer, lung cancer, and prostate cancer. ${ }^{20}$ The US FDA approved it for the treatment of locally advanced or metastatic breast cancer, head and neck cancer, gastric cancer, hormone-refractory prostate cancer, and non-small-cell lung cancer. However, poor water solubility $(6-7 \mu \mathrm{g} / \mathrm{mL})$ remains a critical limitation of DTX. PTX, with a similar structure, also has poor water 
solubility $(\sim 0.3 \mu \mathrm{g} / \mathrm{mL}) .{ }^{21}$ DTX is commercially marketed as Taxotere ${ }^{\circledR}$ or Docefrez ${ }^{\circledR} \cdot 22$ Taxotere contains dehydrated alcohol $(0.395 \mathrm{mg} / \mathrm{mL})$ to improve the solubility of DTX.

Numerous types of NPs have been developed to deliver DTX at the target site, including superparamagnetic iron oxide NPs (SPION), ${ }^{23}$ targeting peptide-conjugated PLA NPs, ${ }^{24}$ polydopamine-modified TPGS-PLA NPs, ${ }^{25}$ cholic acid-functionalized block copolymer consisting of PLGA and vitamin E TPGS, ${ }^{26}$ folic acid-functionalized SLN, ${ }^{27}$ Solutol nanodroplets, ${ }^{28}$ chitosan NPs, ${ }^{29}$ and DTX nanocrystals (DTX-NCs). ${ }^{30}$

Recently, significant advancement has been made in the field of drug NCs because of numerous studies on the development of NCs of drugs such as DTX, ${ }^{30}$ PTX, ${ }^{31}$ curcumin, ${ }^{32}$ aceclofenac, ${ }^{15}$ valsartan, ${ }^{33}$ and quercetin. ${ }^{34}$ Drug NCs are usually prepared by two methods, top-down and bottom-up methods. ${ }^{35,36}$ In the top-down method, the particle size of drugs is reduced by high-pressure homogenization or milling. ${ }^{37-39}$ On the other hand, in the bottom-up method, particle size is controlled by the probe sonication method; furthermore, particle size growth is inhibited by using antisolvents. ${ }^{40,41}$ The preparation method of top-down method is as follows: first, presuspension was prepared by a homogenizer and then the presuspension was processed using a high-pressure homogenizer or microfluidizer. The presuspension consists of micron-sized drug crystals, their particle size was reduced to nanosized drug crystals by a high-pressure homogenizer or microfluidizer. ${ }^{42,43}$ However, the preparation method of bottom-up method is similar to that of the presuspension process in the top-down method with only change of homogenizer to probe sonicator. The drug is fully dissolved in an organic solvent and added dropwise to an antisolvent (aqueous solution), and then increasing size of the drug was inhibited by a probe sonicator. ${ }^{44,45}$

Transferrin is widely used for the surface modification of NPs and for tumor targeting. ${ }^{46,47}$ The cells express transferrin receptors that interact with transferrin of the delivery system. The higher expression of this receptor on cancer cells, its ability to internalize, and the requirement of iron for cancer cell growth make this receptor a widely accessible portal for drug delivery. ${ }^{48,49}$

This study aimed to prepare the DTX-NCs by a nanoprecipitation method (bottom-up). In order to optimize the particle size, several universal buffers were used in the $\mathrm{pH}$ range of 1-10. Most of the drug NCs are surface modified owing to their low stability in aqueous solutions. Therefore, the surface of DTX-NCs was modified with Tf to enhance their stability and tumor targeting. Tf-DTX-NCs were characterized for physical properties, such as morphology and particle size, and in vitro drug release. Moreover, the cytotoxicity and cellular uptake of Tf-DTX-NCs and DTX-NCs were investigated and compared in A549 cells.

\section{Materials and methods Materials}

DTX was obtained from Korea United Pharm. Inc. Co. Ltd. (Seoul, Korea) apo-Transferrin human (Tf), 3-(4,5-di-methylthiazol-2-yl)-2,5-diphenyltetrazolium bromide (MTT), and 4',6-diamidino-2-phenylindole (DAPI) were purchased from Sigma-Aldrich Co (Saint Louis, MO, USA). A549 cells were obtained from the College of Pharmacy, Chungnam National University (supplied by Korean Cell Line Bank, Seoul, Korea). Fetal bovine serum (FBS), antibiotics, and Roswell Park Memorial Institute (RPMI) 1640 medium were obtained from Gibco (Billings, MT, USA). Organic solvents and universal buffers were purchased from Samchun Pure Chemicals (Pyeongtae, Korea).

\section{Methods}

\section{Preparation of DTX-NCs}

DTX-NCs were prepared by the nanoprecipitation method. ${ }^{31}$ Briefly, DTX (4 mg) was dissolved in ethanol (1 mL) and added to universal buffer ( $\mathrm{pH} 1-10 ; 10 \mathrm{~mL}$ ), which was used as an antisolvent, under probe sonication ( $200 \mathrm{~W}$ for 5 minutes with a stirring rate of $300 \mathrm{rpm}$ ) using a probe sonicator (VCX 500; Sonics \& Materials Inc, Newtown, CT, USA) to control the particle size. DTX-NCs were collected by centrifugation at 12,000 rpm for 30 minutes (Avanti ${ }^{\circledR} \mathrm{J}-\mathrm{E}$; Beckman Coulter, Miami, FL, USA) and dried under vacuum in a desiccator for 1 day. All DTX-NCs were stored in a refrigerator at $4^{\circ} \mathrm{C}$.

Tf-DTX-NCs were prepared by the following method: DTX-NCs $(3 \mathrm{mg})$ were redispersed in Tf solution $(0.5 \mathrm{mg} / \mathrm{mL}$ in distilled water [DW]) with a stirring rate of $300 \mathrm{rpm}$ for 1 hour. This suspension was washed with DW and centrifuged two times at 12,000 rpm for 20 minutes (CF-10, Pro-microcentrifuge; DAIHAN Scientific Co., Ltd., Seoul, Korea) and then redispersed in DW (1 mL).

For cellular uptake study, coumarin-6 solution $(100 \mu \mathrm{L}$; $0.1 \mathrm{mg} / \mathrm{mL}$ in ethanol) was added to the DTX-NC suspension (with or without Tf) with a stirring rate of $300 \mathrm{rpm}$ for 1 hour. Coumain-6-adsorbed DTX-NCs and Coumain-6-adsorbed Tf-DTX-NCs were collected by centrifugation at $12,000 \mathrm{rpm}$ for 20 minutes and were redispersed in DW (1 mL).

\section{Morphological characterization by field emission scanning electron microscope (FE-SEM)}

Morphological evaluation of DTX-NCs and Tf-DTX-NCs was carried out using an FE-SEM (JSM-6700F, JEOL, Tokyo, Japan). 
DTX-NCs and Tf-DTX-NCs dispersion were dropped onto a carbon tape and coated with gold for 2 minutes under vacuum. Samples were viewed under an acceleration voltage of $5.0 \mathrm{kV}$. The particle size of at least 50 particles was measured manually using ImageJ software (National Institutes of Health) and averaged to obtain the mean particle size.

\section{In vitro drug release}

In vitro drug release studies were performed in phosphatebuffered saline (PBS; pH 7.4) containing Tween $80(0.5 \%$ w/v) for 24 hours. DTX-NCs, Tf-DTX-NCs, and DTX (pure; equivalent to $50 \mu \mathrm{g} / \mathrm{mL}$ of DTX) were added to the $15 \mathrm{~mL}$ conical tube containing $10 \mathrm{~mL}$ of PBS ( $\mathrm{pH} 7.4)$ containing Tween $80(0.5 \% \mathrm{w} / \mathrm{v})$. The test conditions were a shaking speed of $50 \mathrm{rpm}$ and a temperature of $37^{\circ} \mathrm{C} \pm 0.5^{\circ} \mathrm{C}$. One milliliter of each sample was withdrawn at $0.5,1,2,4,8$, and 24 hours, respectively. The samples were centrifuged at $12,000 \mathrm{rpm}$ for 30 minutes and the supernatants were collected. The DTX content of the samples was determined using high-performance liquid chromatography (HPLC).

\section{HPLC analysis}

DTX content in the samples was analyzed by HPLC (Waters 2695 Alliance system; Waters Corporation, Milford, MA, USA). The mobile phase consisted of acetonitrile and water (70:30). The chromatographic conditions were as follows: an ultraviolet detector (Waters 2487, Waters Corporation) set at $230 \mathrm{~nm}$; a C18 column (Gemini $5 \mu \mathrm{m} \mathrm{110A}$ analytical; Intersil OSD-3, Phenomenex, Torrance, CA, USA) operated at $30^{\circ} \mathrm{C} \pm 1.0^{\circ} \mathrm{C}$; and a flow rate of $1 \mathrm{~mL} / \mathrm{min}$.

\section{Cellular uptake in A549 cells}

A549 cells were grown in RPMI 1640 medium supplemented with $10 \%(\mathrm{v} / \mathrm{v}) \mathrm{FBS}$ and $5 \%$ antibiotics (100 IU/ $\mathrm{mL}$ of penicillin $\mathrm{G}$ sodium and $100 \mu \mathrm{g} / \mathrm{mL}$ of streptomycin sulfate).

The cellular internalization of DTX-NCs and Tf-DTXNCs was visualized by confocal microscopy using coumarin-6 as a fluorescent probe. A549 cells were seeded in a cell culture dish at an initial density of $4 \times 10^{5}$ cells per dish. Cells were then incubated with coumarin-6-adsorbed DTX-NCs or Tf-DTX-NCs (equivalent to $0.1 \mu \mathrm{g} / \mathrm{mL}$ of coumarin-6) for 2 hours at $37^{\circ} \mathrm{C} \pm 0.5^{\circ} \mathrm{C}$. Subsequently, cells were washed several times with PBS and fixed with $4 \%$ paraformaldehyde for 10 minutes. The nuclei were stained with DAPI $(5 \mu \mathrm{g} / \mathrm{mL})$. Finally, cells were observed under a confocal microscope (Eclipse Ti; Nikon, Tokyo, Japan).

For quantitative estimation of DTX uptake, cells were seeded in $24-w e l l$ plates at a density of $3 \times 10^{4}$ cells. When they reached $70 \%-80 \%$ confluence, cells were incubated with coumarin-6-adsorbed DTX-NCs or Tf-DTX-NCs (equivalent to $0.1 \mu \mathrm{g} / \mathrm{mL}$ of coumarin-6). After incubation for $0.5,1$, or 2 hours, cells were washed several times with cold PBS. Subsequently, cells were lysed by addition of Triton X-100 (0.1\%). Fluorescence intensities were measured using a multimode microplate reader (Synergy H1 Hybrid Multi-Mode Microplate Reader; BioTek, Winooski, VT, USA) at an excitation wavelength of $440 \mathrm{~nm}$ and an emission wavelength of $520 \mathrm{~nm}$. Protein contents were quantified using a bicinchoninic acid assay kit (BCA) assay kit (Thermo Fisher Scientific, Waltham, MA, USA), and the fluorescence intensities were normalized.

\section{Cytotoxicity study}

The cytotoxicity of DTX (pure), DTX-NCs, and Tf-DTXNCs was evaluated in A549 cells by MTT assay. Briefly, A549 cells were seeded in a 96-well plate at a density of $3-4 \times 10^{3}$ cells per well. After 12 hours, DTX-NCs and Tf-DTX-NCs (equivalent to DTX concentrations ranging from $1 \mathrm{ng} / \mathrm{mL}$ to $100 \mu \mathrm{g} / \mathrm{mL}$ ) were added, and plates were incubated for 24, 48, and 72 hours. DTX (pure) standard solution was prepared as first DTX (pure) dissolved in ethanol ranging from 0.1 to $10 \mathrm{mg} / \mathrm{mL}$ and then 100 times diluted with DW.

Cytotoxicity was evaluated by MTT assay. Measurements were taken using a microplate reader (Synergy H1 Hybrid Multi-Mode Microplate Reader; BioTek).

\section{Statistical analysis}

Statistical analysis was performed by the paired $t$-test using SigmaPlot 10.0 (SYSTAT, Inc., Chicago, IL, USA). The data are expressed as mean \pm standard deviation. Statistical significance was accepted for $P$-values $<0.05$.

\section{Results and discussion Preparation and optimization of Tf-DTX-NCs}

A nanoprecipitation method was used and optimized to prepare DTX-NCs. The size reduction of DTX was achieved by using universal buffers in the $\mathrm{pH}$ range of $1-10$ and probe sonication. It was hypothesized that DTX is not a pH-dependent drug; however, when DTX solution (DTX dissolved in ethanol) was added to the several $\mathrm{pH}$ universal buffers, the solubility of DTX might be different. As the results suggest, the particle size was almost over $450 \mathrm{~nm}$ under $\mathrm{pH}$ 7 and over $400 \mathrm{~nm}$ above $\mathrm{pH}$ 9; the small particle size was prepared at pH 8 (around $300 \mathrm{~nm}$ ). Moreover, the power of probe sonication was evaluated in the range of 100-500 W. 
As per the results, the particle size was increased depending on increased power probably because of the increased temperature in DTX suspension during probe sonication (data not shown). The optimal parameters were $\mathrm{pH} 8$ and $200 \mathrm{~W}$. Other $\mathrm{pH}$ values of universal buffer and DW were not suitable for preparation of uniform DTX-NCs.

The particle size was measured from the FE-SEM images by using ImageJ software. The mean particle size of DTXNCs and Tf-DTX-NCs was 320.1 $\pm 92.0 \mathrm{~nm}$ (Figure 1A) and $395.6 \pm 101.0 \mathrm{~nm}$ (Figure 1B), respectively. The results of particle size in zetasizer (Nano-90, Malvern Instruments Ltd., Malvern, UK) were $405.0 \pm 101.5 \mathrm{~nm}$ (polydispersity index [PDI]: 0.18) and 468.6 $\pm 120.8 \mathrm{~nm}$ (PDI: 0.18) for DTX-NCs and Tf-DTXNCs, respectively (Table 1). Tf-DTX-NCs exhibited a larger particle size than DTX-NCs owing to the adsorption of Tf on
DTX-NCs. After 7 days, the particle size of DTX-NCs increased (405.3 $\pm 113.0 \mathrm{~nm}$; Figure 1C). Interestingly, Tf-DTX-NCs also showed similar particle size after 7 days $(401.6 \pm 96.0 \mathrm{~nm}$; Figure 1D). The results of particle size in zetasizer were 531.3 $\pm 121.6 \mathrm{~nm}$ (PDI: 0.23) and 480.3 $\pm 107.8 \mathrm{~nm}$ (PDI: 0.17) for DTX-NCs and Tf-DTX-NCs, respectively (Table 1). These results indicate that Tf may have acted as a stabilizer for DTXNCs. In previous studies, it was shown that Tf can also act as a stabilizer for curcumin NPs. ${ }^{32,50}$

The BCA assay kit (Fisher Scientific) was used to quantitatively evaluate the surface modification of DTX-NCs. After surface modification of DTX-NCs with Tf, the supernatant was collected by centrifugation to measure the protein (Tf) concentration. The initial concentration of $\mathrm{Tf}$ solution was $0.5 \mathrm{mg} / \mathrm{mL}$, and final Tf concentration in the Tf-DTX-NCs
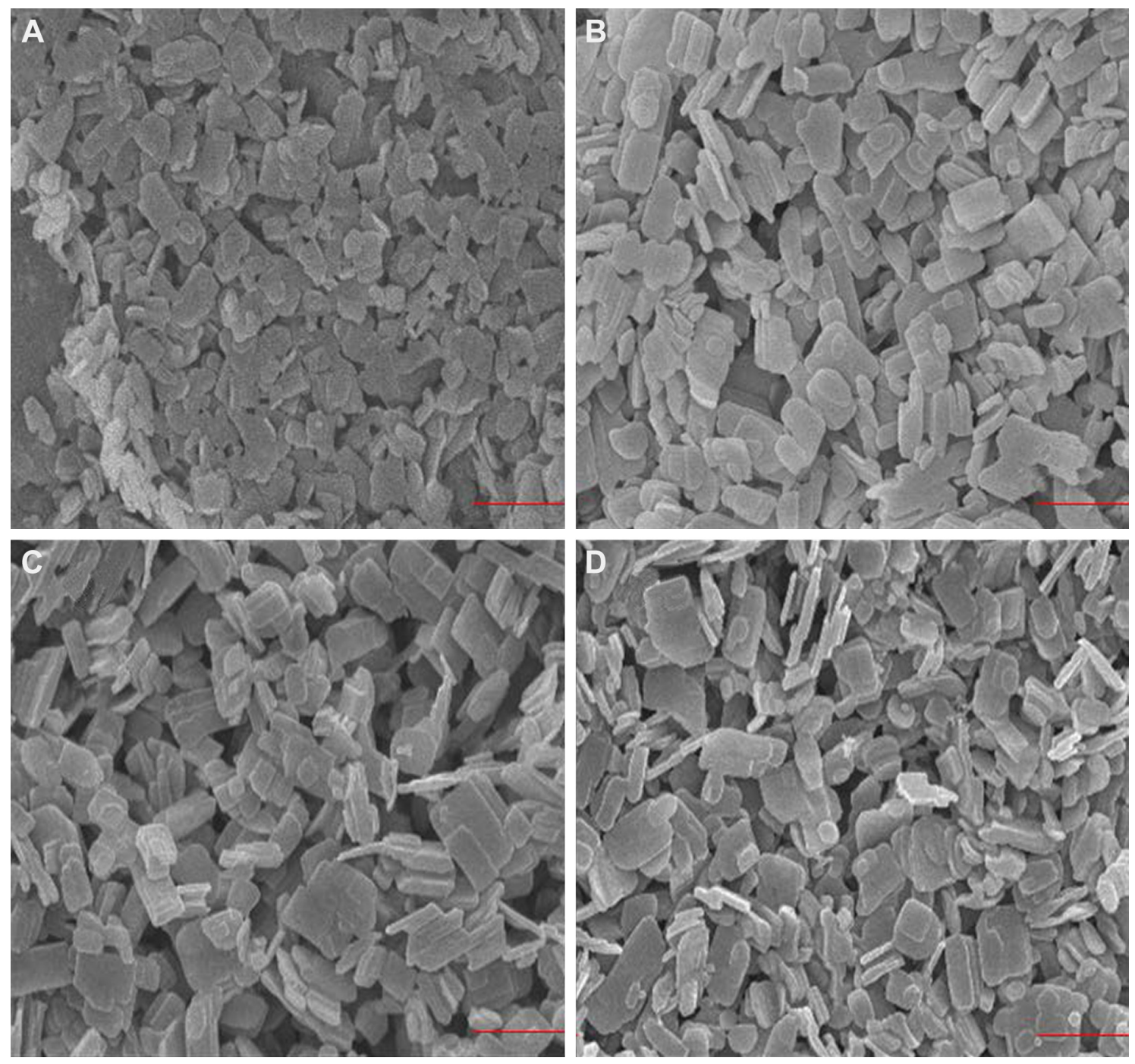

Figure I SEM images.

Notes: SEM images of (A) DTX-NCs, (B) Tf-DTX-NCs, (C) DTX-NC after 7-day storage in DW, and (D) Tf-DTX-NCs after 7-day storage in DW. The magnification is 20,000X; scale bar $=I \mu \mathrm{m}$.

Abbreviations: SEM, scanning electron microscope; DTX, docetaxel; NC, nanocrystal; Tf, apo-Transferrin human; DW, distilled water. 
Table I Physical properties of DTX-NCs and Tf-DTX-NCs (mean \pm SD)

\begin{tabular}{llll}
\hline Properties & Days & DTX-NCs & Tf-DTX-NCs \\
\hline Particle size & Initial day & $320.1 \pm 92.0 \mathrm{~nm}$ & $395.6 \pm 101.0 \mathrm{~nm}$ \\
(SEM) & After 7 days & $405.3 \pm 113.0 \mathrm{~nm}$ & $401.6 \pm 96.0 \mathrm{~nm}$ \\
Particle size & Initial day & $405.0 \pm 101.5 \mathrm{~nm}$ & $468.6 \pm 120.8 \mathrm{~nm}$ \\
(zetasizer) & & (PDI: 0.18$)$ & (PDI: 0.18$)$ \\
& After 7 days & $531.3 \pm 121.6 \mathrm{~nm}$ & $480.3 \pm 107.8 \mathrm{~nm}$ \\
& & (PDI: 0.23$)$ & (PDI: 0.17$)$ \\
\hline
\end{tabular}

Abbreviations: DTX, docetaxel; NCs, nanocrystals; Tf, apo-Transferrin human SEM, scanning electron microscope; PDI, polydispersity index.

was $0.28 \mathrm{mg} / \mathrm{mL}$ (initial concentration of Tf solution $[0.5 \mathrm{mg} / \mathrm{mL}]$ - concentration of Tf remaining in the superna$\operatorname{tant}[0.22 \mathrm{mg} / \mathrm{mL}])$. This confirmed that Tf was successfully adsorbed on the DTX-NCs. Moreover, the stability test of Tf on Tf-DTX-NCs was confirmed by the abovementioned method. Tf-DTX-NC suspension in DW was stored at $4{ }^{\circ} \mathrm{C}$ for 1 week. After 1 week, Tf-DTX-NC suspension was centrifuged and then the supernatant was evaluated. The initial amounts of Tf on Tf-DTX-NCs with that of after 1 week were not changed. This evidence indicated that Tf on Tf-DTX-NCs was stable at $4^{\circ} \mathrm{C}$ for 1 week.

\section{In vitro drug release}

The in vitro drug release of DTX (pure), DTX-NCs, and Tf-DTX-NCs was evaluated in PBS containing Tween 80 $(0.5 \% \mathrm{w} / \mathrm{v})$ for 24 hours. The samples were withdrawn at $0.5,1,2,4,8$, and 24 hours. The DTX cumulative release from the Tf-DTX-NCs was significantly higher than that of DTX (pure) and from DTX-NCs. The cumulative release for DTX (pure), DTX-NCs, and Tf-DTX-NCs at 0.5 hours was

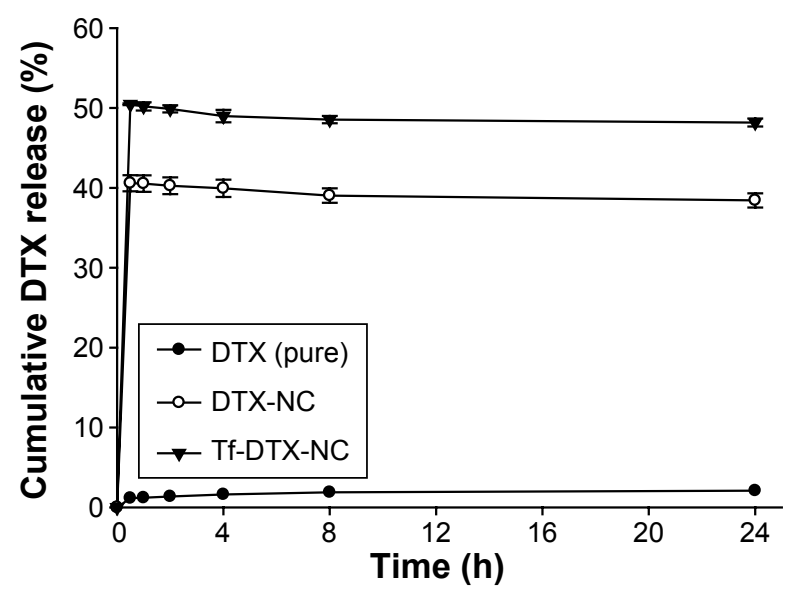

Figure 2 In vitro drug release.

Notes: Drug release of DTX (pure), DTX-NCs, and Tf-DTX-NCs was evaluated in PBS ( $\mathrm{pH} 7.4)$ containing Tween $80(0.5 \% \mathrm{w} / \mathrm{v})$ in a shaking incubator for 24 hours at $37^{\circ} \mathrm{C}$.

Abbreviations: DTX, docetaxel; NC, nanocrystal; Tf, apo-Transferrin human; PBS, phosphate-buffered saline; h, hour.
$1.15 \%, 40.5 \%$, and $50.4 \%$, respectively, following which it reached a steady state (Figure 2). DTX (pure) showed poor drug release because of its poor water solubility.

In previous studies, surface-modified drug crystals showed faster drug release than pure drugs did. ${ }^{15,51,52}$ Drug NCs have smaller size than pure drug. They have an increased dissolution rate because of the decreased diffusion distance. ${ }^{53}$ Furthermore, water-soluble materials like $\mathrm{Tf}$ are believed to increase the wetting ability of drug NCs.

\section{Cellular uptake}

A qualitative cellular uptake study was performed by using confocal microscopy (Figure 3). Cell internalization in A549 cells was higher for the surface-modified DTX-NCs than DTX-NCs. Results showed that depending on surface modification, cell internalization was changed.

For quantitative cellular uptake study, coumarin- 6 on the DTX-NCs and Tf-DTX-NCs was quantified by recovering the drug NCs from cells and measuring their fluorescence (normalized to per milligram of the total cellular protein contents). The results of quantification were in agreement with the confocal images. DTX-NCs and Tf-DTX-NCs exhibited similar results at short incubation time ( 0.5 and 1 hour). However, at longer incubation time (2 hours), Tf-DTX-NCs showed higher cellular uptake than DTX-NCs did (Figure 4). The results of Tf-DTX-NCs were significantly different $(P<0.01$; paired $t$-test) from those obtained for DTX-NCs at all incubation times, except at 0.5 hour $(P=0.02)$.

In a previous study, Tf-adsorbed PLGA NPs exhibited higher uptake than BSA-adsorbed PLGA NPs did in F98 cells (brain cancer cells) ${ }^{54}$ Similarly, Tf-LPs (Tf surface-modified lipid-coated PLGA NPs) showed higher uptake than modified lipid-coated PLGA NPs (LPs) in A549 cells. ${ }^{55}$ The enhanced DTX cellular uptake in this study confirmed the targeting ability of Tf-DTX-NCs.

\section{Cytotoxicity study}

The cytotoxicity of Tf-DTX-NCs and DTX-NCs was evaluated in A549 cancer cells. The cytotoxicity of DTX (pure), DTX-NCs, and Tf-DTX-NCs was evaluated at eight-point concentrations and three-point times (Figure 5). NC samples also were redispersed in DW to obtain the final concentrations ranging from $1 \mathrm{ng} / \mathrm{mL}$ to $100 \mu \mathrm{g} / \mathrm{mL}$ (equivalent DTX concentration). The drug content of the samples was determined by HPLC.

For 24-hour incubation, Tf-DTX-NCs $(66.9 \% \pm 3.8 \%)$ showed higher cytotoxicity than DTX-NCs $(55.5 \% \pm 6.1 \%)$ and DTX (pure; $15.5 \% \pm 5.7 \%$ ) at a DTX concentration of $100 \mu \mathrm{g} / \mathrm{mL}$, which was 1.2 and 4.3 times higher than that 


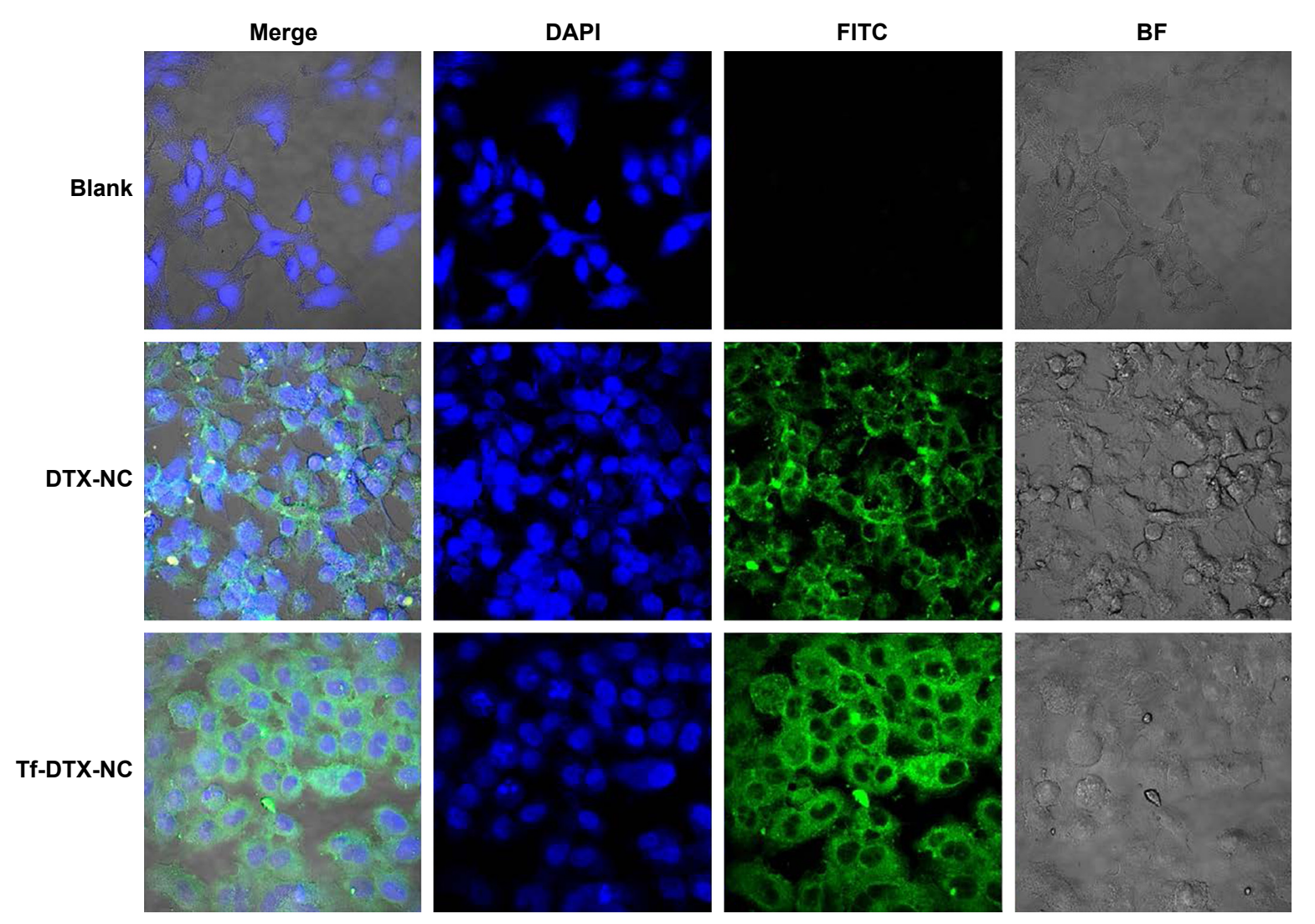

Figure 3 Confocal images.

Notes: Confocal images of transferrin-dependent cellular uptake of Tf-DTX-NC by A549 cells. Incubation time was 2 hours. Green and blue colors represent courmarin-6-adsorbed Tf-DTX-NCs or DTX-NCs, and nucleus (DAPI), respectively.

Abbreviations: Tf, apo-Transferrin human; DTX, docetaxel; NC, nanocrystal; DAPI, 4',6-diamidino-2-phenylindole; FITC, fluorescein isothiocyanate; BF, bright field.

of DTX-NCs and DTX (pure), respectively. For 48-hour incubation, Tf-DTX-NCs $(75.1 \% \pm 1.9 \%)$ showed higher cytotoxicity than DTX-NCs $(61.9 \% \pm 3.1 \%)$ and DTX (pure; $18.6 \% \pm 3.0 \%$ ) at a DTX concentration of $100 \mu \mathrm{g} / \mathrm{mL}$, which was 1.2 and 4.3 times higher than that of DTX-NCs and DTX (pure), respectively. For 72-hour incubation, Tf-DTX-NCs

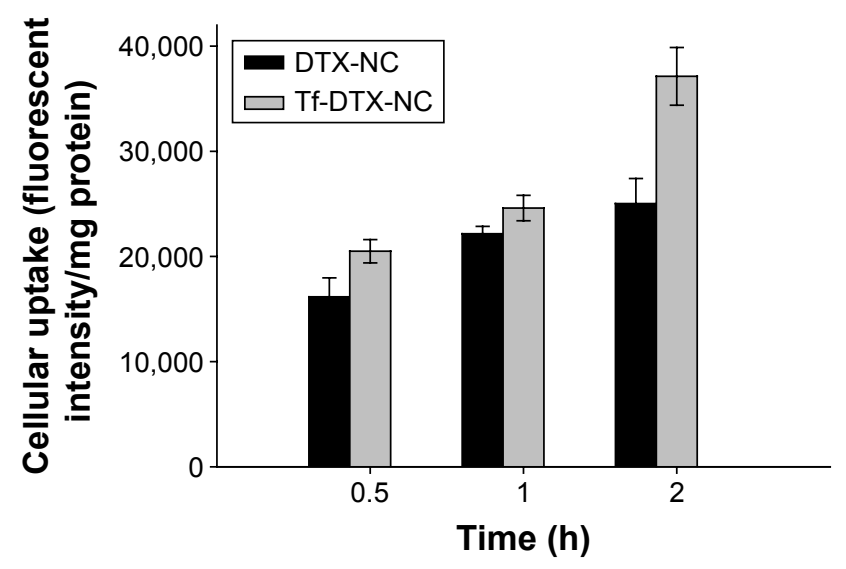

Figure 4 Quantification of the cellular uptake.

Note: Data are expressed as mean $\pm S D(n=4)$.

Abbreviations: DTX, docetaxel; NC, nanocrystal; Tf, apo-Transferrin human; h, hour.
$(82.6 \% \pm 0.8 \%)$ showed higher cytotoxicity than DTX-NCs $(77.4 \% \pm 4.1 \%$ ) and DTX (pure; $20.1 \% \pm 4.6 \%$ ) at a DTX concentration of $100 \mu \mathrm{g} / \mathrm{mL}$, which was 1.1 and 4.1 times higher than that of DTX-NCs and DTX (pure), respectively. All of the concentrations and times showed greater cytotoxicity of Tf-DTX-NCs compared to other groups. In particular, the cytotoxicity of DTX (pure) increased until $25 \mu \mathrm{g} / \mathrm{mL}$ and then decreased. During the preparation of DTX standard solution, solutions changed the color from clear to turbid at 50 and $100 \mu \mathrm{g} / \mathrm{mL}$. For this reason, when DTX ethanol solution was diluted with water, the drug was precipitated due to differences in solubility. Microscopic analysis showed the presence of micronized DTX which was due to the precipitation (data not shown). The half maximal inhibitory concentration (IC50) values were $13.7 \pm 0.6 \mu \mathrm{g} / \mathrm{mL}$ (DTX-NCs) and $4.8 \pm 0.7 \mu \mathrm{g} / \mathrm{mL}$ (Tf-DTX-NCs) at 24-hour incubation time, 2.9 times less than DTX-NCs. At 48-hour incubation time, IC50 values were $3.9 \pm 0.5 \mu \mathrm{g} / \mathrm{mL}$ (DTX-NCs) and 1.6 $\pm 0.4 \mu \mathrm{g} / \mathrm{mL}$ (Tf-DTX$\mathrm{NCs}$ ), which was 2.4 times less than DTX-NCs. IC50 values were $1.0 \pm 0.5 \mu \mathrm{g} / \mathrm{mL}$ (DTX-NCs) and $0.5 \pm 0.3 \mu \mathrm{g} / \mathrm{mL}$ (Tf-DTX-NCs) at 72-hour incubation time, two times less than 

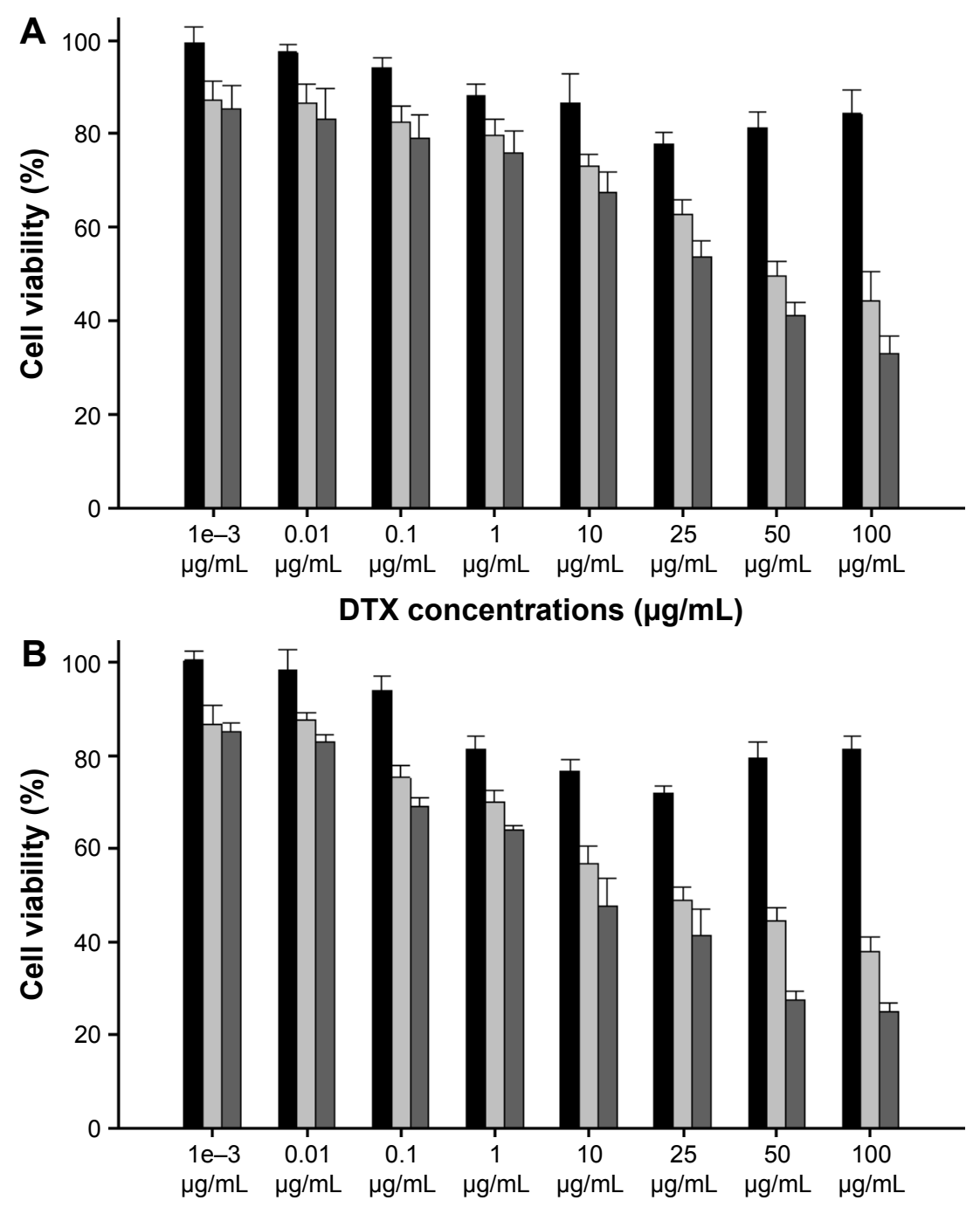

DTX concentrations $(\mu \mathrm{g} / \mathrm{mL})$

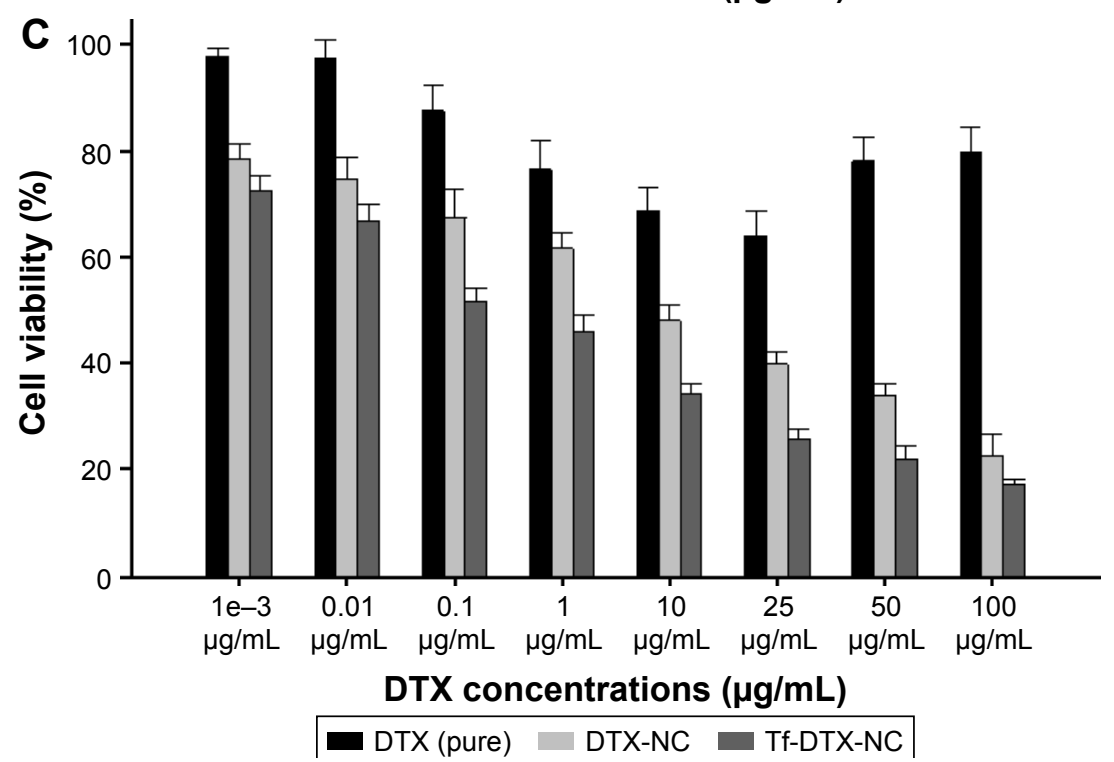

Figure 5 Evaluation of cytotoxicity.

Notes: Cytotoxicity of DTX (pure) and DTX-NCs or Tf-DTX-NCs was compared at 24 hours (A), 48 hours (B), and 72 hours (C). Drug concentrations ranged from I $\mathrm{ng} / \mathrm{mL}$ to $100 \mu \mathrm{g} / \mathrm{mL}$.

Abbreviations: DTX, docetaxel; NC, nanocrystal; Tf, apo-Transferrin human. 
Table 2 IC50 values of DTX-NCs and Tf-DTX-NCs in A549 cells following 24-, 48-, and 72-hour treatments, respectively $(n=6)$

\begin{tabular}{lll}
\hline $\begin{array}{l}\text { Incubation } \\
\text { time (hours) }\end{array}$ & $\begin{array}{l}\text { DTX-NCs } \\
(\mu \mathrm{g} / \mathrm{mL})\end{array}$ & $\begin{array}{l}\text { Tf-DTX-NCs } \\
(\mu \mathrm{g} / \mathrm{mL})\end{array}$ \\
\hline 24 & $13.7 \pm 0.6$ & $4.8 \pm 0.7$ \\
48 & $3.9 \pm 0.5$ & $1.6 \pm 0.4$ \\
72 & $1.0 \pm 0.5$ & $0.5 \pm 0.3$ \\
\hline
\end{tabular}

Abbreviations: DTX, docetaxel; NCs, nanocrystals; Tf, apo-Transferrin human.

the DTX-NCs. Thus, IC50 value was reduced depending on incubation times (Table 2).

The results of the cytotoxicity assay for all the samples were significantly different from those obtained for DTX (pure) at all concentrations (paired $t$-test; $P<0.05$ ).

Many studies have demonstrated that the surfacemodified NPs or NCs have higher cytotoxicity than the surface-unmodified NPs, NCs, or pure drugs ${ }^{56-58}$ The results of this study showed that Tf-DTX-NCs have strong targeting ability, which can improve the cellular uptake as well as cytotoxicity of DTX.

\section{Conclusion}

DTX-NCs were prepared by the nanoprecipitation method and were surface modified with Tf (Tf-DTX-NCs). The obtained Tf-DTX-NCs were in the nanosize range required to invade cancer cells. Tf-DTX-NCs were more stable and showed faster DTX release than DTX-NCs and DTX (pure). Moreover, Tf-DTX-NCs significantly enhanced the cellular uptake and cytotoxicity of DTX in A549 cell line. In vivo studies with Tf-DTX-NCs will be undertaken in future to evaluate and confirm the efficacy of this $\mathrm{NC}$ system.

\section{Acknowledgment}

This research was supported by Basic Science Research Program through the National Research Foundation of Korea (NRF) funded by the Korean government, MSIP (No 2015R1A2A1A10051596), and MOE (No 20090093815).

\section{Disclosure}

The authors report no conflicts of interest in this work.

\section{References}

1. Xiao D, Jia HZ, Zhang J, Liu CW, Zhuo RX, Zhang XZ. A dualresponsive mesoporous silica nanoparticle for tumor-triggered targeting drug delivery. Small. 2014;10(3):591-598.

2. Chen T, Zhao T, Wei D, Wei Y, Li Y, Zhang H. Core-shell nanocarriers with $\mathrm{ZnO}$ quantum dots-conjugated $\mathrm{Au}$ nanoparticle for tumor-targeted drug delivery. Carbohydr Polym. 2013;92(2):1124-1132.

3. Sreeja S, Nair CKK. Chemo-directed specific targeting of nanoparticledoxorubicin complexes to tumor in animal model. J Drug Deliv Sci Technol. 2016;31:167-175.
4. Jose S, Sowmya S, Cinu T, Aleykutty N, Thomas S, Souto E. Surface modified PLGA nanoparticles for brain targeting of Bacoside-A. Eur J Pharm Sci. 2014;63:29-35.

5. Maeda H, Nakamura H, Fang J. The EPR effect for macromolecular drug delivery to solid tumors: improvement of tumor uptake, lowering of systemic toxicity, and distinct tumor imaging in vivo. Adv Drug Deliv Rev. 2013;65(1):71-79.

6. Steichen SD, Caldorera-Moore M, Peppas NA. A review of current nanoparticle and targeting moieties for the delivery of cancer therapeutics. Eur J Pharm Sci. 2013;48(3):416-427.

7. Zhong Y, Goltsche K, Cheng L, et al. Hyaluronic acid-shelled acid-activatable paclitaxel prodrug micelles effectively target and treat CD44overexpressing human breast tumor xenografts in vivo. Biomaterials. 2016;84:250-261

8. Sheng Z, Song L, Zheng J, et al. Protein-assisted fabrication of nanoreduced graphene oxide for combined in vivo photoacoustic imaging and photothermal therapy. Biomaterials. 2013;34(21):5236-5243.

9. Jalalian SH, Taghdisi SM, Hamedani NS, et al. Epirubicin loaded super paramagnetic iron oxide nanoparticle-aptamer bioconjugate for combined colon cancer therapy and imaging in vivo. Eur J Pharm Sci. 2013;50(2): 191-197.

10. Tamba B, Dondas A, Leon M, et al. Silica nanoparticles: preparation, characterization and in vitro/in vivo biodistribution studies. Eur J Pharm Sci. 2015;71:46-55.

11. Choi J-S, Cao J, Naeem M, et al. Size-controlled biodegradable nanoparticles: preparation and size-dependent cellular uptake and tumor cell growth inhibition. Colloids Surf B Biointerfaces. 2014;122:545-551.

12. Nabi-Meibodi M, Navidi B, Navidi N, Vatanara A, Rouini MR, Ramezani V. Optimized double emulsion-solvent evaporation process for production of solid lipid nanoparticles containing baclofene as a lipid insoluble drug. J Drug Deliv Sci Technol. 2013;23(3):225-230.

13. Lv Q, Li L-M, Han M, et al. Characteristics of sequential targeting of brain glioma for transferrin-modified cisplatin liposome. Int J Pharm. 2013;444(1):1-9.

14. She W, Luo K, Zhang C, et al. The potential of self-assembled, pHresponsive nanoparticles of mPEGylated peptide dendron-doxorubicin conjugates for cancer therapy. Biomaterials. 2013;34(5):1613-1623.

15. Park J-J, Meghani N, Choi J-S, Lee B-J. Development and evaluation of decorated aceclofenac nanocrystals. Colloids Surf B Biointerfaces. 2016; 143:206-212.

16. Le Broc-Ryckewaert D, Carpentier R, Lipka E, et al. Development of innovative paclitaxel-loaded small PLGA nanoparticles: study of their antiproliferative activity and their molecular interactions on prostatic cancer cells. Int J Pharm. 2013;454(2):712-719.

17. Choi J-S, Meghani N. Impact of surface modification in BSA nanoparticles for uptake in cancer cells. Colloids Surf B Biointerfaces. 2016; 145:653-661.

18. Yu K, Zhao J, Zhang Z, et al. Enhanced delivery of Paclitaxel using electrostatically-conjugated Herceptin-bearing PEI/PLGA nanoparticles against HER-positive breast cancer cells. Int J Pharm. 2016; 497(1):78-87.

19. $\mathrm{Hu} \mathrm{Q}, \mathrm{Gu} \mathrm{G}$, Liu Z, et al. F3 peptide-functionalized PEG-PLA nanoparticles co-administrated with tLyp-1 peptide for anti-glioma drug delivery. Biomaterials. 2013;34(4):1135-1145.

20. Du N, Song L-P, Li X-S, et al. Novel pH-sensitive nanoformulated docetaxel as a potential therapeutic strategy for the treatment of cholangiocarcinoma. J Nanobiotechnology. 2015;13(1):17.

21. Soga O, van Nostrum CF, Fens M, et al. Thermosensitive and biodegradable polymeric micelles for paclitaxel delivery. J Control Release. 2005;103(2):341-353.

22. Blumenthal GM, Scher NS, Cortazar P, et al. First FDA approval of dual anti-HER2 regimen: pertuzumab in combination with trastuzumab and docetaxel for HER2-positive metastatic breast cancer. Clin Cancer Res. 2013;19(18):4911-4916.

23. Nagesh PK, Johnson NR, Boya VK, et al. PSMA targeted docetaxelloaded superparamagnetic iron oxide nanoparticles for prostate cancer. Colloids Surf B Biointerfaces. 2016;144:8-20. 
24. Jiang Y, Yang N, Zhang H, et al. Enhanced in vivo antitumor efficacy of dual-functional peptide-modified docetaxel nanoparticles through tumor targeting and Hsp90 inhibition. J Control Release. 2016;221:26-36.

25. Zhu D, Tao W, Zhang H, et al. Docetaxel (DTX)-loaded polydopaminemodified TPGS-PLA nanoparticles as a targeted drug delivery system for the treatment of liver cancer. Acta Biomater. 2016;30:144-154.

26. Zeng X, Tao W, Mei L, Huang L, Tan C, Feng S-S. Cholic acidfunctionalized nanoparticles of star-shaped PLGA-vitamin E TPGS copolymer for docetaxel delivery to cervical cancer. Biomaterials. 2013; 34(25):6058-6067.

27. Pawar H, Surapaneni SK, Tikoo K, et al. Folic acid functionalized long-circulating co-encapsulated docetaxel and curcumin solid lipid nanoparticles: in vitro evaluation, pharmacokinetic and biodistribution in rats. Drug Deliv. 2016;23(4):1453-1468.

28. Oh KS, Kim K, Yoon BD, et al. Docetaxel-loaded multilayer nanoparticles with nanodroplets for cancer therapy. Int J Nanomedicine. 2016; $11: 1077$.

29. Jain A, Thakur K, Sharma G, Kush P, Jain UK. Fabrication, characterization and cytotoxicity studies of ionically cross-linked docetaxel loaded chitosan nanoparticles. Carbohydr Polym. 2016;137: 65-74.

30. Pawar VK, Gupta S, Singh Y, et al. Pluronic F-127 stabilised docetaxel nanocrystals improve apoptosis by mitochondrial depolarization in breast cancer cells: pharmacokinetics and toxicity assessment. J Biomed Nanotechnol. 2015;11(10):1747-1763.

31. Choi J-S, Park J-S. Effects of paclitaxel nanocrystals surface charge on cell internalization. Eur J Pharm Sci. 2016;93:90-96.

32. Choi J-S. Development of surface curcumin nanoparticles modified with biological macromolecules for anti-tumor effects. Int J Biol Macromol. 2016;92:850-859.

33. Biswas N, Kuotsu K. Chronotherapeutically modulated pulsatile system of valsartan nanocrystals - an in vitro and in vivo evaluation. AAPS Pharm Sci Tech. Epub 2016 Mar 9.

34. Lai F, Franceschini I, Corrias F, et al. Maltodextrin fast dissolving films for quercetin nanocrystal delivery. A feasibility study. Carbohydr Polym. 2015;121:217-223.

35. Gao L, Liu G, Ma J, et al. Application of drug nanocrystal technologies on oral drug delivery of poorly soluble drugs. Pharm Res. 2013; 30(2):307-324.

36. Guo M, Fu Q, Wu C, et al. Rod shaped nanocrystals exhibit superior in vitro dissolution and in vivo bioavailability over spherical like nanocrystals: a case study of lovastatin. Colloids Surf B Biointerfaces. 2015; 128:410-418.

37. Chen H, Khemtong C, Yang X, Chang X, Gao J. Nanonization strategies for poorly water-soluble drugs. Drug Discov Today. 2011;16(7):354-360.

38. Merisko-Liversidge E, Liversidge GG. Nanosizing for oral and parenteral drug delivery: a perspective on formulating poorly-water soluble compounds using wet media milling technology. Adv Drug Deliv Rev. 2011;63(6):427-440.

39. Yi Y, Tu L, Hu K, Wu W, Feng J. The construction of puerarin nanocrystals and its pharmacokinetic and in vivo-in vitro correlation (IVIVC) studies on beagle dog. Colloids Surf B Biointerfaces. 2015; 133:164-170.

40. Ige PP, Baria RK, Gattani SG. Fabrication of fenofibrate nanocrystals by probe sonication method for enhancement of dissolution rate and oral bioavailability. Colloids Surf B Biointerfaces. 2013;108:366-373.

41. Pattekari P, Zheng Z, Zhang X, Levchenko T, Torchilin V, Lvov Y. Top-down and bottom-up approaches in production of aqueous nanocolloids of low solubility drug paclitaxel. Phys Chem Chem Phys. 2011; 13(19):9014-9019.
42. Sun W, Mao S, Shi Y, Li LC, Fang L. Nanonization of itraconazole by high pressure homogenization: stabilizer optimization and effect of particle size on oral absorption. J Pharm Sci. 2011;100(8):3365-3373.

43. Hao L, Wang X, Zhang D, et al. Studies on the preparation, characterization and pharmacokinetics of Amoitone B nanocrystals. Int J Pharm. 2012;433(1):157-164.

44. Xia D, Quan P, Piao H, et al. Preparation of stable nitrendipine nanosuspensions using the precipitation-ultrasonication method for enhancement of dissolution and oral bioavailability. Eur J Pharm Sci. 2010;40(4):325-334.

45. Verma S, Gokhale R, Burgess DJ. A comparative study of top-down and bottom-up approaches for the preparation of micro/nanosuspensions. Int J Pharm. 2009;380(1):216-222.

46. Cui Y, Xu Q, Chow PK-H, Wang D, Wang C-H. Transferrin-conjugated magnetic silica PLGA nanoparticles loaded with doxorubicin and paclitaxel for brain glioma treatment. Biomaterials. 2013; 34(33):8511-8520.

47. Liu T, Kempson I, de Jonge M, Howard DL, Thierry B. Quantitative synchrotron X-ray fluorescence study of the penetration of transferrinconjugated gold nanoparticles inside model tumour tissues. Nanoscale. 2014;6(16):9774-9782.

48. Ponka P, Lok CN. The transferrin receptor: role in health and disease. Int J Biochem Cell Biol. 1999;31(10):1111-1137.

49. Xu Q, Liu Y, Su S, Li W, Chen C, Wu Y. Anti-tumor activity of paclitaxel through dual-targeting carrier of cyclic RGD and transferrin conjugated hyperbranched copolymer nanoparticles. Biomaterials. 2012;33(5):1627-1639.

50. Yadav D, Kumar N. Nanonization of curcumin by antisolvent precipitation: process development, characterization, freeze drying and stability performance. Int J Pharm. 2014;477(1):564-577.

51. Li Y, Wu Z, He W, et al. Globular protein-coated paclitaxel nanosuspensions: interaction mechanism, direct cytosolic delivery, and significant improvement in pharmacokinetics. Mol Pharm. 2015;12(5): 1485-1500.

52. Sharma S, Verma A, Teja BV, Shukla P, Mishra PR. Development of stabilized paclitaxel nanocrystals: in-vitro and in-vivo efficacy studies. Eur J Pharm Sci. 2015;69:51-60.

53. Chu KR, Lee E, Jeong SH, Park E-S. Effect of particle size on the dissolution behaviors of poorly water-soluble drugs. Arch Pharm Res. 2012;35(7):1187-1195.

54. Chang J, Paillard A, Passirani C, et al. Transferrin adsorption onto PLGA nanoparticles governs their interaction with biological systems from blood circulation to brain cancer cells. Pharm Res. 2012;29(6):1495-1505.

55. Guo Y, Wang L, Lv P, Zhang P. Transferrin-conjugated doxorubicinloaded lipid-coated nanoparticles for the targeting and therapy of lung cancer. Oncol Lett. 2015;9(3):1065-1072.

56. Manoochehri S, Darvishi B, Kamalinia G, et al. Surface modification of PLGA nanoparticles via human serum albumin conjugation for controlled delivery of docetaxel. Daru. 2013;21(1):58.

57. Kulkarni SA, Feng S-S. Effects of particle size and surface modification on cellular uptake and biodistribution of polymeric nanoparticles for drug delivery. Pharm Res. 2013;30(10):2512-2522.

58. Lu Y, Wang Z-H, Li T, McNally H, Park K, Sturek M. Development and evaluation of transferrin-stabilized paclitaxel nanocrystal formulation. J Control Release. 2014;176:76-85. 


\section{Publish your work in this journal}

Drug Design, Development and Therapy is an international, peerreviewed open-access journal that spans the spectrum of drug design and development through to clinical applications. Clinical outcomes, patient safety, and programs for the development and effective, safe, and sustained use of medicines are the features of the journal, which

has also been accepted for indexing on PubMed Central. The manuscript management system is completely online and includes a very quick and fair peer-review system, which is all easy to use. Visit http://www.dovepress.com/testimonials.php to read real quotes from published authors.

Submit your manuscript here: http://www.dovepress.com/drug-design-development-and-therapy-journal 